\title{
FPGA IMPLEMENTATION OF MASS PUBLIC TRANSIT FACILITY FOR SMART SECURITY SYSTEM
}

\author{
M. Prakash ${ }^{1}$, K. Vijaipriya ${ }^{2}$, S. Nandhini ${ }^{3}$ \\ ${ }^{1} P G$ Scholar, Department of ECE, Ranganathan Engineering College, Coimbatore, India \\ ${ }^{2}$ Assistant Professor, Department of ECE, Ranganathan Engineering College, Coimbatore, India \\ ${ }^{3}$ PG Scholar, Department of ECE, Ranganathan Engineering College, Coimbatore, India
}

\begin{abstract}
With the increasing number of bus users, many initiatives have been undertaken to enhance customer's satisfaction. The RFIDbased luggage tracking system would make the process of luggage handling easier and faster. We are going to fix RF Transmitter into the luggages, once the destination is reached, and then he/she should enter the user ID. If the code matches with database then the luggage is delivered to corresponding passenger. In case of lost or mishandled luggage, the alarm will be activated and the information is passed to the control station and the message will be sent to the passenger regarding their luggage status via GSM communication. For the security tendency of the vehicle is improved by scanning the person entering into the bus using Gas sensor. If a person is suspected of carrying any illegal materials, then the buzzer will be activated and the information is displayed. To ensure the security features, we have introduced alert switches nearby every seat. Whenever the switch is pressed, automatically the vehicle will be turned off and all the lights will be turned on and high alarm is activated so that the public can interfere and avoid the misbehavior. At the same time, the information contains a bus number or an ID will be sent to the Police station using SMS facility. All these processes are controlled by an FPGA controller with the high speed of processing using Xilinx ISE.
\end{abstract}

Keywords- FPGA, RFID, GSM Communication, Gas sensor, SMS

\section{INTRODUCTION}

Nowadays the number of bus users is increasing and many initiatives have been undertaken to enhance customer's satisfaction. The public transit is a transport service for passengers to share the travel along different places, as distinct from models such as taxicabs or hired buses which are not shared by strangers without private arrangement. Public transits include city buses, passenger trains and other transits. The RFID transceivers are implemented for luggage tracking system in buses. The use of an interactive RFIDbased luggage tracking system would make the process of baggage handling easier and faster [1]. At present, the use of barcode system for baggage and passenger transport, which is an old and traditional method with high error percentages. In this system, transport operation is conducted at very low speed and precision. The currently used baggage handling system causes a large number of mishandled bags. Mishandled baggage generates big losses to companies and individuals.

Also, the security tendency of the vehicle is improved by scanning the person entering into the bus. By this method, we can track the terrorist activities in city buses and stop their illegal activities. If a person is suspected of carrying any illegal materials then the Alarms will be activated, at the same time the information will be passed to the police station through GSM Communication with bus ID [2]. Finally, to ensure the security features of women travelling in public transit, we have introduced Alert switches nearby every seat. Whenever the switch is activated automatically vehicle will be turned off and all the lights will be turned on and high alarm will be activated.
The main objective is to achieve the security system in public transport and avoidance of women attacks in real time process. By the means of this process, the vehicle security has been increased and easy to find the lost luggage, avoids the illegal materials in the public space, also we exclude the misbehaviors. Here SPARTAN3AN FPGA [5] is used as the main processor to make the system flexible and process it in parallel manner.

\section{RELATED WORK}

Mostafa Tavassoli, Abolfazl Rajabi, Mehrdad Javadi, Sasan Mohammadi [1] proposed the work to increase the efficiency of the baggage which carried among the public. In this, unique number has been given to the each passenger who is all carrying the luggages. By the use of unique number to each luggage's thereby the efficiency has been improved and passengers mismatch will be avoided.

Afif Mghawish, Akram Abdel Qader, Mohammad AbuMahfouz [2] proposes the distributed multi control in wireless networks. For this multi control applications, the GSM communication has been used to improve the security tendency of home automation. The system is tested and configured to set an alarm through the mobile phone using SMS facility.

Wael M ElMedany, Mahmoud R ElSabry [3] introduced the 24 hours of user friendly for monitoring the realtime and remote sensing system. This will be designed and implemented with the FPGA and it programmed completely 
with VHDL. By updating the AT commands, the main control unit separates the messages according to the number of devices used in it. The security has been increased by this updating process.

Fengxiang Qiao, Lei Yu, Rong Zhang, Zhiyuan Chen, Reza Fatholahzadeh [4] uses RFID to improve the vehicle safety and security. The RFID transfers the information between the passenger and their luggage. Using this process, the luggage status will be updated every moment and improves the human lives in the intelligent transportation system. Also it save cost, safety driving and will reduce vehicle emission and collision.

Yadav Vajrangshakti.Ramdayal, K.Srinivasa Rao [5] describes the security system in the automation industries. The temperature and gas monitoring system has been introduced to concentrate more on safety precautions. Here Spartan3an is used with the GSM communication to improve the security.

\section{GENERAL BLOCK DIAGRAM}
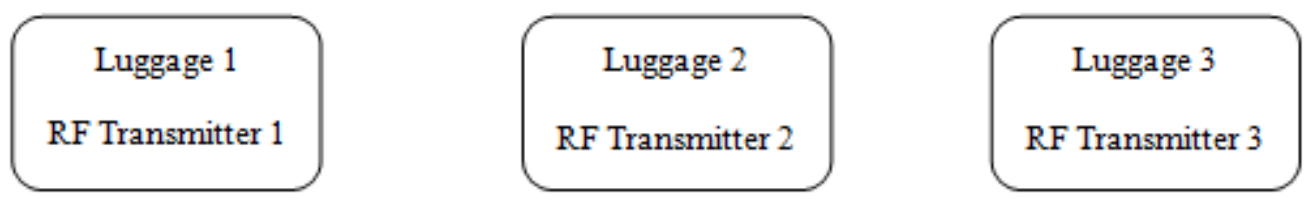

Fig -1: In the Luggage Section

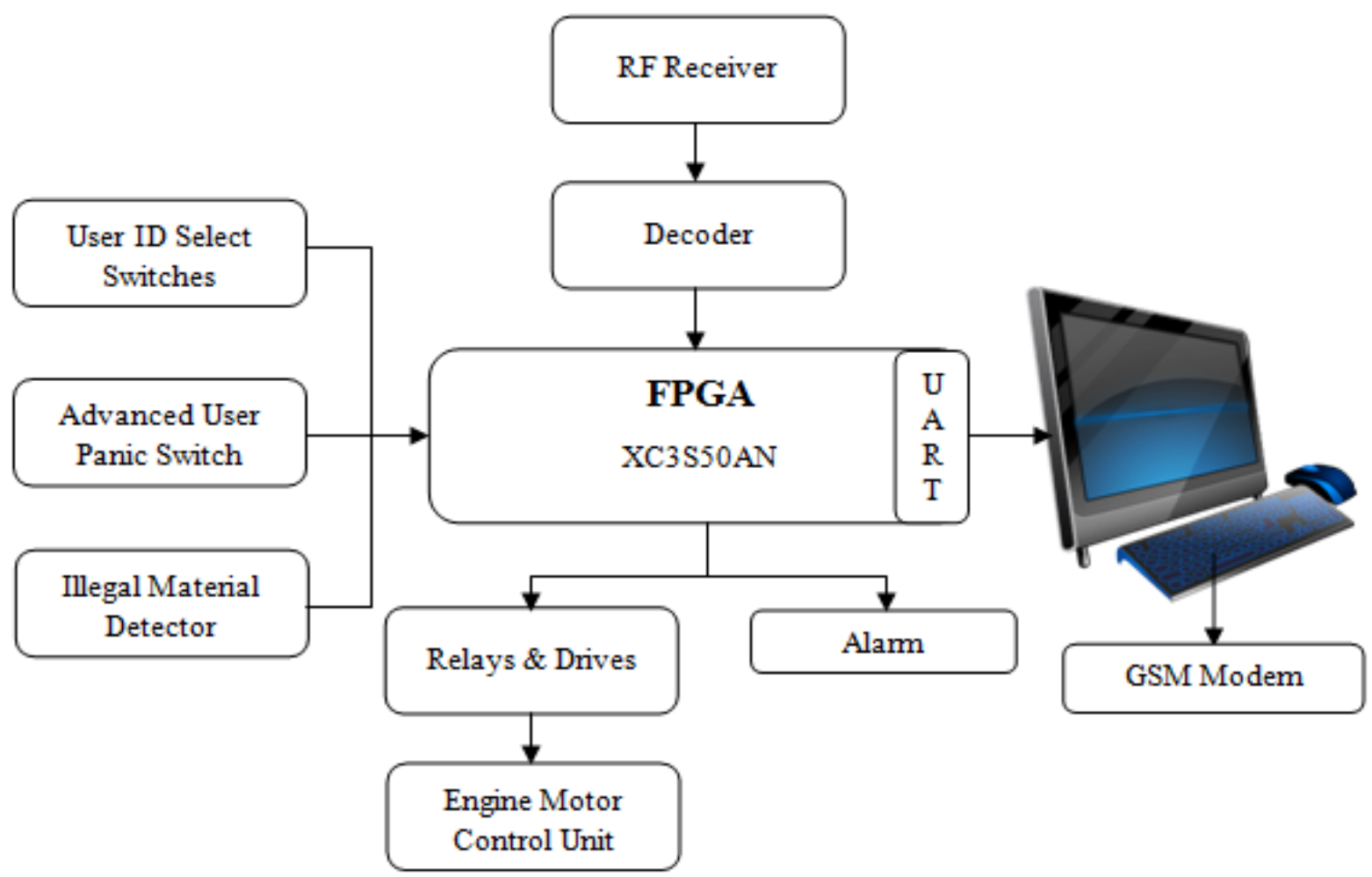

Fig -2: Block diagram in the Bus section

From the figure 1, consider the three luggage's with the fixed RFID. Each luggage has separate RF transmitter which means unique number have been defined. Passenger should aware of his/her luggage unique number. The figure 2 shows the general block diagram with FPGA Spartan3AN as a main controller and its being connected with the other modules.

\section{HARDWARE MODULES}

\subsection{FPGA (Field Programmable Gate Array)}

The term "field-programmable" means the device is completely programmed by the user. The manufacturer will not responsible for the programming device. It can be programmed using a logic circuit diagram or source code in VHDL or Verilog. An FPGA is a reprogrammable chip which contains hundreds of thousands of logic gates that internally connects together to build complex digital circuitry. It offers partial re-configuration of a portion of design. 
The Spartan-3AN FPGA family is a low cost FPGA with nonvolatile technology. Spartan-3AN FPGAs are programmed by loading configuration data into static CMOS configuration latches (CCLs) that control all elements and resources. Some features are integrated robust configuration memory, Saves board space, Improves easeof-use, Simplifies design and Reduces support issues. Xilinx Spartan-3AN family XC3S50AN FPGA device is offered in only one package and it as standard TQG144 package.

\subsection{Radio Frequency Transmitter/Receiver}

The RFID transmitter/receiver modules are used to deliver luggage's to their respective passengers. The luggage's safety and secured using this RFID and mishandled will be stopped. This RF transmitter module is consists of small printed circuit board capable of transmitting radio waves and this modulated wave carries an data. Normally transmitter modules are implemented with the microcontroller which will transmit the provided data to the module. The RF receiver receives the modulated signal from the transmitter and demodulates it. The receiver antenna is quite simple and it operates in the few microvolts signal level.

\subsection{Gas Sensor}

The Gas Sensor type MQ2 used in our project to detect the illegal materials carried in a public transits. It is used to sense the presence of gas in the surrounding areas. The gases like flammable, combustible and toxic gases are initially detected. If any gases have been detected then the alarm will be turned on. Gas sensor is a subclass of chemical sensors and it measures the vicinity concentration of gas. The device which measures the current discharge of pre determined concentration of gas. Common gases measured by gas sensors include ammonia, carbon dioxide, carbon monoxide, chlorine, chlorine dioxide, fluorine, hydrogen, hydrogen chloride, hydrogen cyanide, nitrogen dioxide, nitrogen oxides, nitric oxide, organic solvents, oxygen, ozone, sulfur dioxide and water vapor. Some characteristic configurations are Good sensitivity to Combustible gas in wide range, High sensitivity, Long life and low cost and it has simple drive circuit.

\subsection{Panic Switch}

To ensure the security features in the public transportation, the panic switch have been introduced. From the Spartan3AN, the one slide switch is act as a panic switch as sample. By pressing this switch automatically the vehicle will be turned off and the lights are turned on, correspondingly the information is passed to the control unit. Also the alarm is turned on if the switch is pressed. For the realtime process, the switch is implemented nearby every seat in the vehicle. Using this implementation, the passengers travelling in the vehicle can easily press the switch if any misbehavior attempted.

\subsection{GSM Module}

If any mishandling or misbehavior occurred in the vehicle then GSM module is used send the information or message to the control unit. Here we have used the GSM/GPRS Modem-RS232 is built with Dual Band GSM/GPRS engineSIM900A. The SIM900A is a complete Quad-band GSM/GPRS solution in a SMT module which can be embedded in the customer applications. The RS232 cable is used to interface module with PC and with main controller. The external $9 \mathrm{~V}$ supply has to be used to operate the module.

The GSM/GPRS Modem is having internal TCP/IP stack to enable you to connect with internet via GPRS. It is suitable for SMS, Voice as well as DATA transfer application in M2M interface. Using this modem, you can make audio calls, SMS, Read SMS; attend the incoming calls and internet act through simple AT commands. These kinds of GSM modules are used in varied places to send and receive the information from different places. It used in Access control devices and Supply chain management. 


\section{EXPERIMENTAL RESULTS}

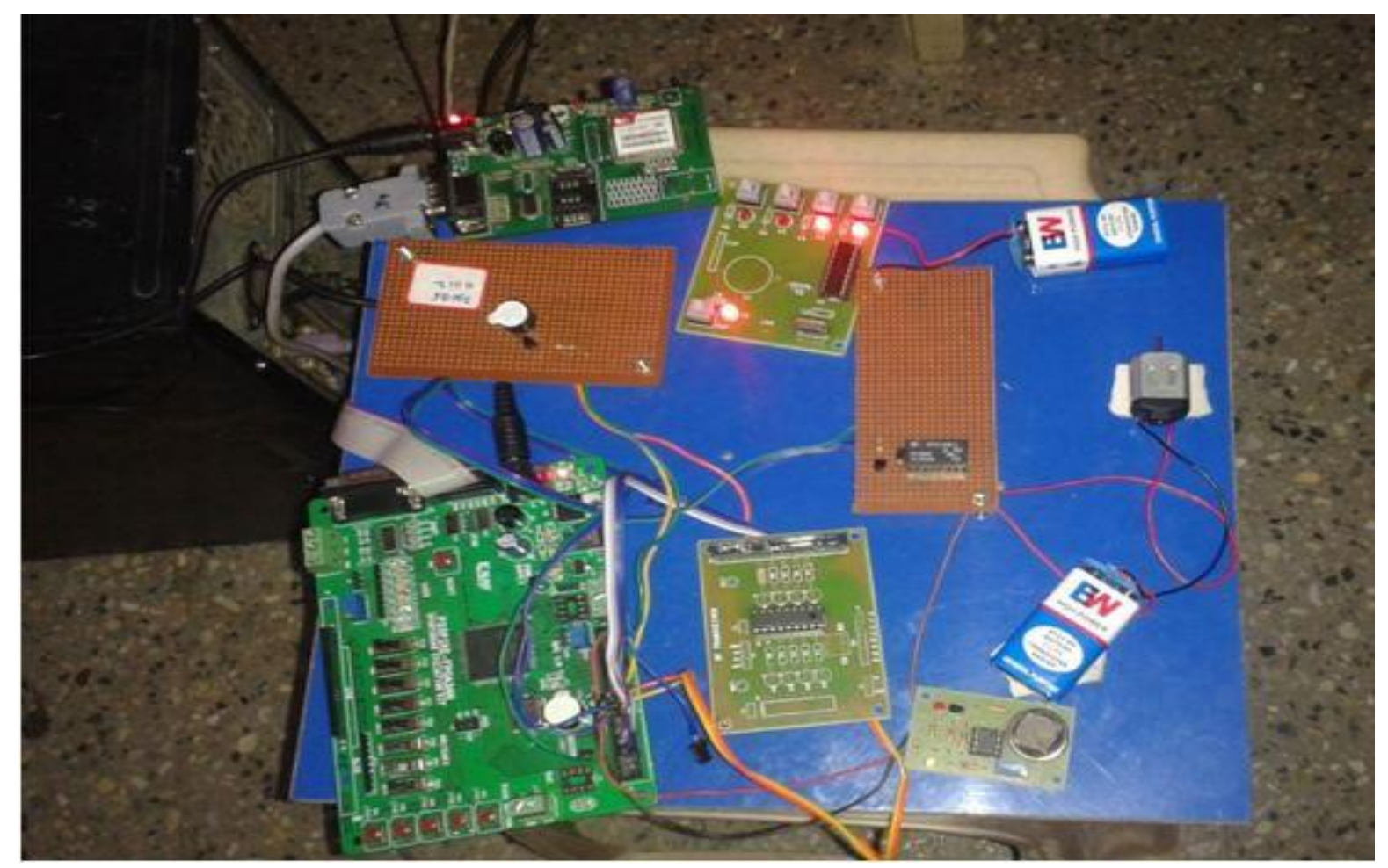

Fig -3: Hardware view of Bus module

The figure 3 shows the hardware view of bus module. From this figure, the modules FPGA Spartan3AN, GSM modem, RF transmitter and receiver, Gas sensor, buzzer, motor and relay are interfaced each other. Here FPGA Spartan3AN $\mathrm{XC3S50AN}$ is used as a main controller. This module requires RS232 and Parallel cable to interface with PC. Using the Xilinx software, VHDL coding have been programmed to initialize number of $\mathrm{I} / \mathrm{O}$ pins used and defining the corresponding values to interface other modules. The VHDL program is created as per the general block diagram requirements and its being dumped into the FPGA module using the JTAG port. Once the program has any syntax or compilation error then it be cleared and checked again. The user constrain file is created to assign the pins which are used to connect other external modules. After the VHDL program is dumped into the FPGA kit, the done led will blow to indicate that program successfully implemented into the kit. As per the user constrain file created, external module pins are connected with the main controller. The Modules FPGA and GSM modem requires external power supply of $9 \mathrm{~V}$ to switch on the kit.

The RF transmitter requires a $5 \mathrm{~V}$ battery to operate the circuit board. The 4 switches are configured as passenger's identification code and its being previously initialized in VHDL program. The RF receiver data and voltage pins are connected with the FPGA kit as the pre-programmed configuration. If the user A switch is pressed in the transmitter then the modulated signal is received at the receiver and it demodulates. The demodulated signal is processed in the main controller and checks the information of passenger whether he/she authenticated to deliver the luggage. If the passenger pressed an incorrect switch or any mishandling occurred then automatically the information is passed through the control station and to the corresponding message to the passengers about their luggage status. Using this manner, the passenger's luggage traveling in the public transportation is secured.

Gas sensor used in our project to detect or sense the presence of gas in public transportation. The sensor data and voltage pins are connected as per the pins initialized in the VHDL program. The gas sensor output pins are interfaced with the FPGA kit to pass the information to the control unit. If the person carrying any illegal material or any other gas solvents inside the public transits then gas is sensed and the information is passed into the control station and corresponding message to the respective security head using GSM modem. The IC LM358 is used for the operation of gas sensor and it has two internally compensated op amps with the need for dual supplies are eliminated.

Additional security feature of panic switch have been introduced to avoid the misbehavior happen to women. Nowadays women's are needed to travel in public transportation along long distance in their day-to-day life. This panic switch will give some safety and security measures to the all passengers which makes journey safer. Here we have consider one slide switch in FPGA kit as panic switch and this completely programmed as the panic action is pressed. If vehicle suspects that panic switch is pressed, then engine motor will be turned off and lights are turned on. The DC motor is considered as the engine motor for a sample and it operates with the relay circuitry. The 
LED lights which are inbuilt in FPGA kit as the lights present in the public transportation. Once the panic switch is pressed then bus ID and the information is passed to control station using GSM communication. The corresponding bus status will be updated through SMS to the owner of the vehicle.

GSM modem is used to communicate the information and vehicle status to the respective control station. This requires
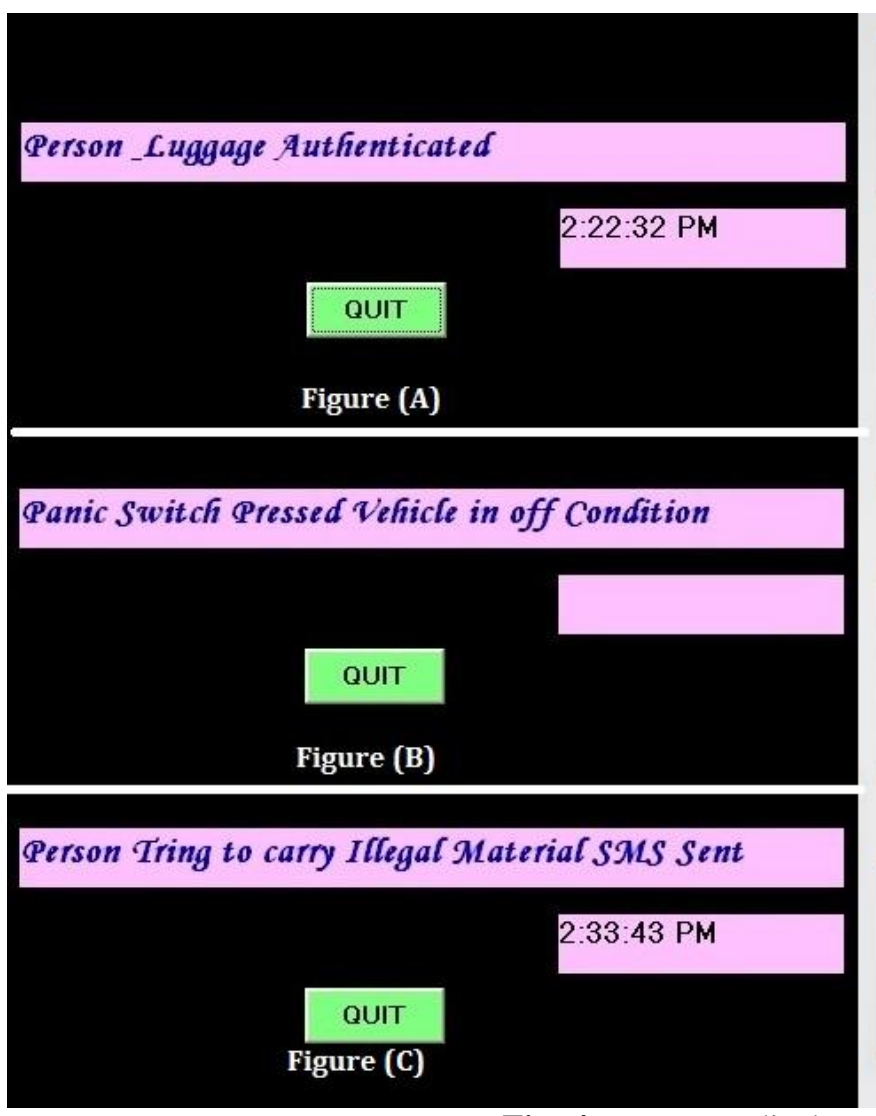

9V supply and RS232 to communicate with the PC. If any mishandling or misbehavior happen in the public transportation then automatically the SMS is sent to the control unit and alarm is constructed of buzzer is turned on. These features are to be concentrated to make the vehicle safety and secure.

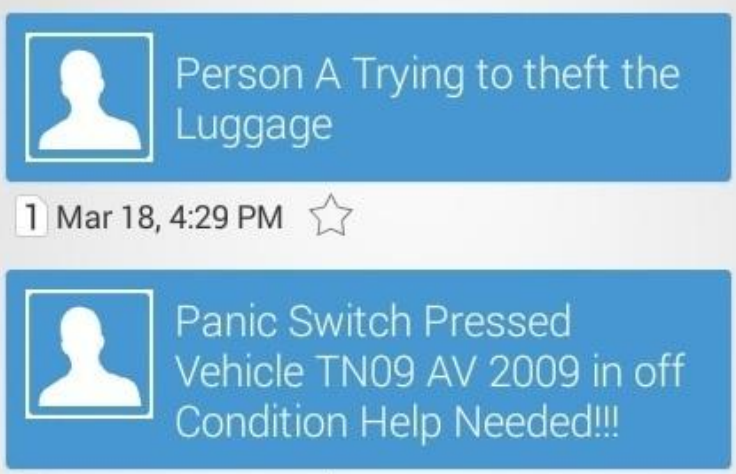

1 Mar 18, 4:29 PM $\widehat{\text { W }}$

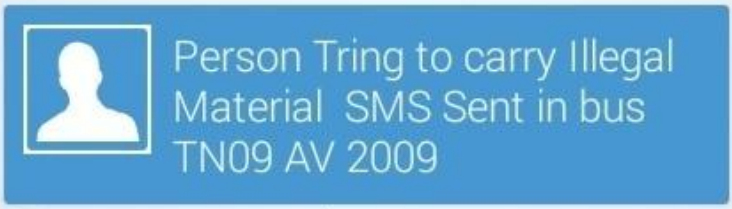

1 Mar 18, 4:30 PM

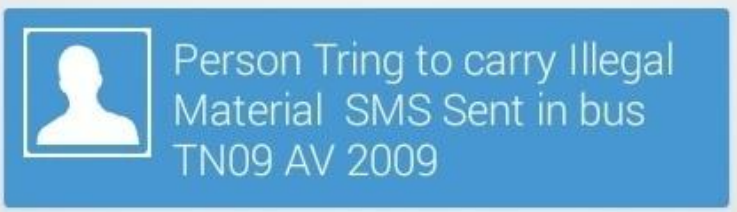

Figure (D)

Fig -4: Messages displayed in control station

From the above figure, each figure A, B, C and D represents the different messages. By pressing the different keys, the messages have been displayed to the control station. The figure $\mathrm{A}, \mathrm{B}$ and $\mathrm{C}$ are information displayed in the control station and figure $\mathrm{D}$ represents the list of messages received from the vehicle through GSM communication. The figure A shows the person is authenticated handle the luggage and if any mismatch is happened then the message displayed as person $\mathrm{A}$ is trying to theft the luggage and the corresponding luggage status is sent to the passenger. Next if the vehicle suspect of panic switch is pressed, then information is passed to the control station and through the GSM communication which is represented in figure B. From figure $\mathrm{C}$, if the person detects of carrying any illegal material inside the public transportation then the information is passed to the control station and through the message. The figure $\mathrm{D}$ shows the list messages received from the vehicle about luggage status, women's safety and transportation safety. We should aware of these kinds of security measures during the travel. In case of any emergency requirements, these features are much helpful to achieve the medical help easier and faster. By this passengers life will also be secured when these features implemented in a vehicle.

\section{ADVANTAGES AND APPLICATIONS}

The unique advantages of Spartan-3AN Generation FPGAs across in wide range of applications. Here some advantages are

- $\quad$ FPGA flexibility and scalability

- $\quad$ Field upgradeability

- Fabric parallelism

- $\quad$ Embedded Processing Technology

- $\quad$ Fast Simplex Link and Xtreme DSP Technology

The some applications and their challenges are

- $\quad$ Flat Panel Displays - Shorter product life cycles with higher amortized cost.

- Automotive - Full compliance to production process and quality standards. 
- Industrial Ethernet and Motion Control - Bridging multiple connectivity protocols.

- Video Surveillance - DSP performance need for object recognition, motion detection algorithms.

\section{CONCLUSION}

In public transport system, the implementation of this equipment will ease the people. Passengers are free to go anywhere with this security purpose. If any mishandled is happen then the information is passed through GSM communication and also women can travel in the public transit at anytime. Once the panic switch is pressed, then automatically the bus ID will be sent to both the control and police station. If the person found of carrying any illegal materials, then the information sent to the control station.

\section{FUTURE WORK}

This project can be enhanced in future by modifying the program to find out the actual position of the vehicle using the GPS system. Instead of waiting in bus station, the Zigbee module gives the status/place of bus is reached and the passenger's time have been saved. This can be used to prevent vehicle theft. In case of any theft, the owner can track the location of the vehicle. Thus we can make use of the available technology to the benefit of the people and helping the owners of the vehicle to keep track of their vehicles.

\section{REFERENCES}

[1] Abolfazl Rajabi, Mehrdad Javadi, Mostafa Tavassoli and Sasan Mohammadi (2012), 'Baggage Traffic Control in Airports making use of RFID Technology', IJSCE, vol. 2, no. 5, pp. 111-116.

[2] Afif Mghawish, Akram Abdel Qader and Mohammad AbuMahfouz (2013), 'Distributed Multi Control Monitoring Application (DMCMA) based on Mobile/Wireless Networks', IJCSIT, vol. 1, no. 3, pp. 1-9.

[3] Mahmoud R El-Sabry and Wael M El-Medany (2008), 'GSM-Based Remote Sensing and Control System using FPGA', Proceedings of the International Conference on Computer and Comm Engineering, pp 1093-1097.

[4] Fengxiang Qiao, Lei Yu, Reza Fatholahzadeh, Rong Zhang and Zhiyuan Chen (2009), 'RFID Applications in Transportation Operation and Intelligent Transportation Systems', U.S. Department of Transportation, no. SWUTC/09/476660-00044-1.

[5] Yadav Vajrangshakti.Ramdayal1, K.Srinivasa Rao (2013), 'An Adaptive industrial automation application design using ASIC', IJRRECS, Vol. 1, no. 6, pp. 1093-1099.

[6] Aranguren G., Arias J., Blazquez A., and Nozal L. (2002), 'Remote control of sensors and actuators by GSM', IEEE 28th Annual Conference of the Industrial Electronics Society IECON 02, vol. 3, pp. $2306-2310$.
[7] Arias Tanti Hapsari, Eniman Y Syamsudin and Imron Pramana (2005), 'Design Of Vehicle Position Tracking System using Short Message Services and its Implementation On FPGA', Proceedings of Asia and South Pacific Design Automation Conference, ISBN: 0-7803-8737-6.

[8] Doug Creighton, Michael Johnstone and Saeid Nahavandi (2010), Senior Member, IEEE,'Statusbased Routing in Baggage Handling Systems: Searching Verses Learning', IEEE transactions on systems, man, and cybernetics-PART C: Applications and Reviews, VOL. 40, NO. 2.

[9] Hou Y., Ouyang Y., Pang L., Wang D., and Xiong Z. (2008), 'An intelligent RFID reader and its application in airport baggage handling system', 4th International Conference on Wireless Communications, Networking and Mobile Computing, pp. 1-4. 